\title{
Pseudo-Hermitian versus Hermitian position-dependent-mass Hamiltonians in a perturbative framework
}

\author{
B Bagchi ${ }^{a}$, C Quesne ${ }^{b}$, R Roychoudhury ${ }^{c}$ \\ ${ }^{a}$ Department of Applied Mathematics, University of Calcutta, \\ 92 Acharya Prafulla Chandra Road, Kolkata 700 009, India \\ ${ }^{b}$ Physique Nucléaire Théorique et Physique Mathématique, Université Libre de Bruxelles, \\ Campus de la Plaine CP229, Boulevard du Triomphe, B-1050 Brussels, Belgium \\ ${ }^{c}$ Physics and Applied Mathematics Unit, Indian Statistical Institute, Kolkata 700 035, India \\ E-mail: bbagchi123@rediffmail.com, cquesne@ulb.ac.be,raj@isical.ac.in
}

\begin{abstract}
We formulate a systematic algorithm for constructing a whole class of Hermitian position-dependent-mass Hamiltonians which, to lowest order of perturbation theory, allow a description in terms of $\mathcal{P} \mathcal{T}$-symmetric Hamiltonians. The method is applied to the Hermitian analogue of the $\mathcal{P} \mathcal{T}$-symmetric cubic anharmonic oscillator. A new example is provided by a Hamiltonian (approximately) equivalent to a $\mathcal{P} \mathcal{T}$-symmetric extension of the one-parameter trigonometric Pöschl-Teller potential.
\end{abstract}

Keywords: Schrödinger equation, pseudo-Hermiticity, $P T$-symmetry, position-dependent mass

PACS No.: 03.65.-w 
Pseudo-Hermitian Hamiltonians and their subclass of $\mathcal{P} \mathcal{T}$-symmetric ones have aroused a great deal of interest since it was observed that some of them may have a real, positive spectrum [1]. Pseudo-Hermiticity of $H$ with respect to a positive-definite (Hermitian and invertible) operator $\eta_{+}$, i.e.,

$$
H^{\dagger}=\eta_{+} H \eta_{+}^{-1}
$$

has been identified as one of the necessary and sufficient conditions for this situation to occur 2]. Any Hamiltonian endowed with such a property is then equivalent to a Hermitian one

$$
h=\rho H \rho^{-1}
$$

where the similarity transformation is implemented by $\rho=\sqrt{\eta_{+}}$. Further, to any observable $o$ and to any wavefunction $\psi(x)=\langle x \mid \psi\rangle$ in the Hermitian theory described by $h$, one can associate an operator $O=\rho^{-1} \rho \rho$ and a wavefunction $\Psi(x)=\langle x \mid \rho \psi\rangle$ in the (physical) pseudo-Hermitian theory, respectively.

Recently Jones [3] and, independently, Mostafazadeh [4] constructed the Hermitian analogue $h$, as well as the pseudo-Hermitian position and momentum operators $X=\rho^{-1} x \rho$, $P=\rho^{-1} p \rho$, for the $\mathcal{P} \mathcal{T}$-symmetric cubic anharmonic oscillator $H=\frac{1}{2}\left(p^{2}+x^{2}\right)+\mathrm{i} \epsilon x^{3}$ (with $\epsilon \in \mathbb{R}$ ). The latter, which has been shown both numerically [1] and mathematically [5] to have a real, positive and discrete spectrum, can only be treated in perturbation theory [ 6 . A very interesting outcome of [3] and [4] is that to lowest order such a system describes an ordinary quartic anharmonic oscillator with real and positive coupling constants but a position-dependent mass (PDM). As revealed by a more recent study of Bender et al [7], this Hermitian PDM theory is however difficult to work out because it leads to divergent Feynman graphs, which must be regulated to obtain the correct answer, whereas the corresponding non-Hermitian $\mathcal{P} \mathcal{T}$-symmetric theory is completely free from such difficulties.

At this stage, it is worth mentioning that Hermitian PDM Hamiltonians are attracting a lot of attention due to their relevance in describing the physics of many microstructures of current interest, such as compositionally graded crystals (see [8] and references quoted therein). Several classes of physically-interesting solvable non-Hermitian potentials have also been generated [9, 10, 11] in a PDM background by employing various techniques, 
such as the point canonical transformations or Lie algebraic methods, or using ideas from supersymmetric quantum mechanics. In particular, constructions of $\mathcal{P} \mathcal{T}$-symmetric potentials have been carried out for different choices of mass functions. These include the $\mathcal{P} \mathcal{T}$ symmetric Scarf potential [9] and the $\mathcal{P} \mathcal{T}$-symmetric oscillator model [10]. Even the PDM version of the complex Morse potential [12], which is known to be pseudo-Hermitian [13, has been obtained [10].

In view of all these considerations, it may prove interesting to see under which conditions a Hermitian PDM Hamiltonian may be approximately equivalent to a non-Hermitian $\mathcal{P} \mathcal{T}$ symmetric one, which, according to the experience gained in [7], would presumably be easier to handle. In the spirit of [3] and [4, this is tantamount to determining those $\mathcal{P} \mathcal{T}$-symmetric Hamiltonians,

$$
H=H_{0}+\varepsilon H_{1} \quad H_{0}=\frac{p^{2}}{2 m_{0}}+V^{(\mathrm{r})}(x) \quad H_{1}=\mathrm{i} V^{(\mathrm{i})}(x)
$$

with $\varepsilon \in \mathbb{R}, V^{(\mathrm{r})}(x)=V^{(\mathrm{r})}(-x) \in \mathbb{R}, V^{(\mathrm{i})}(x)=-V^{(\mathrm{i})}(-x) \in \mathbb{R}$ and configuration space $\mathbb{R}$ (or a subset of it), that have a Hermitian counterpart

$$
h=H_{0}+\varepsilon^{2} h^{(2)}+\varepsilon^{4} h^{(4)}+\cdots
$$

which to lowest order in $\varepsilon$ reduces to some PDM Hamiltonian, i.e.,

$$
H_{0}+\varepsilon^{2} h^{(2)}=p \frac{1}{2 m(x)} p+V_{\text {eff }}(x)
$$

with $1 / m(x)=\left(1 / m_{0}\right)\left[1+\varepsilon^{2} M^{(2)}(x)\right], V_{\text {eff }}(x)=V^{(\mathrm{r})}(x)+\varepsilon^{2} V_{\text {eff }}^{(2)}(x)$ and $M^{(2)}(x), V_{\text {eff }}^{(2)}(x) \in$ $\mathbb{R}$. It should be noted that the right-hand side of (4) only contains even powers of $\varepsilon$ because the coefficients of odd powers have been shown to vanish [3, 4], while the right-hand side of (5) is the most general expression of Hermitian PDM Hamiltonians [8]. The latter is written in terms of an effective potential $V_{\text {eff }}(x)$ including some mass terms depending on two ambiguity parameters, which take the noncommutativity of the momentum and PDM operators into account [14].

It proves convenient to introduce dimensionless quantities defined by

$$
\begin{aligned}
& \mathrm{x}=\ell^{-1} x \quad \mathrm{p}=\ell \hbar^{-1} p \\
& \mathrm{H}=\nu^{-1} H=\mathrm{H}_{0}+\varepsilon \mathrm{H}_{1} \quad \mathrm{H}_{0}=\frac{1}{2} \mathrm{p}^{2}+\mathrm{V}^{(\mathrm{r})}(\mathrm{x}) \quad \mathrm{H}_{1}(\mathrm{x})=\mathrm{iV}^{(\mathrm{i})}(\mathrm{x}) \\
& \mathrm{h}=\nu^{-1} h=\mathrm{H}_{0}+\varepsilon^{2} \mathrm{~h}^{(2)}=\frac{1}{2} \mathrm{p}\left[1+\varepsilon^{2} \mathrm{M}^{(2)}(\mathrm{x})\right] \mathrm{p}+\mathrm{V}^{(\mathrm{r})}(\mathrm{x})+\varepsilon^{2} \mathrm{~V}_{\mathrm{eff}}^{(2)}(\mathrm{x})
\end{aligned}
$$


in terms of some length and energy scales, $\ell$ and $\nu=\hbar^{2} /\left(m_{0} \ell^{2}\right)$. Note that in (3), (4) and (5), $\varepsilon$ is also dimensionless, as well as $M^{(2)}(x)$.

In [3] and [4] (see also [6]), it has been shown that for the positive-definite metric operator $\eta_{+}$, one may take

$$
\eta_{+}=e^{-Q(\mathrm{x}, \mathrm{p})} \quad Q(\mathrm{x}, \mathrm{p})=\varepsilon Q_{1}(\mathrm{x}, \mathrm{p})+\varepsilon^{3} Q_{3}(\mathrm{x}, \mathrm{p})+\cdots
$$

where every $Q_{j}(\mathrm{x}, \mathrm{p}), j=1,3, \ldots$, is such that $Q_{j}(\mathrm{x}, \mathrm{p})=Q_{j}^{\dagger}(\mathrm{x}, \mathrm{p})=Q_{j}(-\mathrm{x}, \mathrm{p})=$ $-Q_{j}(\mathrm{x},-\mathrm{p})$. Then to lowest order in $\varepsilon$, equations (11) and (2I) lead to the two conditions

$$
\left[\mathrm{H}_{0}, Q_{1}\right]=-2 \mathrm{H}_{1} \quad \frac{1}{4}\left[\mathrm{H}_{1}, Q_{1}\right]=\mathrm{h}^{(2)}
$$

which in the case of (3) and (15) amount to

$$
\begin{aligned}
& {\left[\frac{1}{2} \mathrm{p}^{2}+\mathrm{V}^{(\mathrm{r})}(\mathrm{x}), Q_{1}\right]=-2 \mathrm{iV}(\mathrm{i})(\mathrm{x})} \\
& \frac{\mathrm{i}}{4}\left[\mathrm{~V}^{(\mathrm{i})}(\mathrm{x}), Q_{1}\right]=\frac{1}{2} \mathrm{pM}^{(2)}(\mathrm{x}) \mathrm{p}+\mathrm{V}_{\mathrm{eff}}^{(2)}(\mathrm{x}) .
\end{aligned}
$$

For $Q_{1}$, let us choose a general ansatz somewhat different from those previously considered:

$$
Q_{1}=\sum_{k=0}^{\infty}\left\{R_{k}(\mathrm{x}), \mathrm{p}^{2 k+1}\right\} \quad R_{k}(\mathrm{x})=R_{k}(-\mathrm{x}) .
$$

By expressing $\mathrm{p}$ as $-\mathrm{i} d / d \mathrm{x}$ and using the commutation relation

$$
\left[\frac{d^{k}}{d \mathrm{x}^{k}}, f(\mathrm{x})\right]=\sum_{l=0}^{k-1}\left(\begin{array}{c}
k \\
l
\end{array}\right) \frac{d^{k-l} f(\mathrm{x})}{d \mathrm{x}^{k-l}} \frac{d^{l}}{d \mathrm{x}^{l}}
$$

$Q_{1}$ can be written in normal form, i.e., with all functions of $\mathrm{x}$ on the left of the differential operators, as

$$
Q_{1}=-\mathrm{i} \sum_{k=0}^{\infty} S_{k}(\mathrm{x}) \frac{d^{k}}{d \mathrm{x}^{k}}
$$

where

$$
\begin{aligned}
& S_{2 k}=\sum_{l=k}^{\infty}(-1)^{l}\left(\begin{array}{c}
2 l+1 \\
2 k
\end{array}\right) \frac{d^{2 l-2 k+1} R_{l}}{d \mathrm{x}^{2 l-2 k+1}} \\
& S_{2 k+1}=\sum_{l=k}^{\infty}\left(1+\delta_{l, k}\right)(-1)^{l}\left(\begin{array}{c}
2 l+1 \\
2 k+1
\end{array}\right) \frac{d^{2 l-2 k} R_{l}}{d \mathrm{x}^{2 l-2 k}}
\end{aligned}
$$


for $k=0,1,2, \ldots$

On inserting (13) in (9) and (10) and employing (12) again, we find after some straightforward calculations that equation (9) is equivalent to the conditions

$$
\begin{aligned}
& \frac{1}{2} \frac{d^{2} S_{0}}{d \mathrm{x}^{2}}+\sum_{l=1}^{\infty} S_{l} \frac{d^{l} \mathrm{~V}^{(\mathrm{r})}}{d \mathrm{x}^{l}}=-2 \mathrm{~V}^{(\mathrm{i})} \\
& \frac{d S_{k-1}}{d \mathrm{x}}+\frac{1}{2} \frac{d^{2} S_{k}}{d \mathrm{x}^{2}}+\sum_{l=k+1}^{\infty}\left(\begin{array}{c}
l \\
k
\end{array}\right) S_{l} \frac{d^{l-k} \mathrm{~V}^{(\mathrm{r})}}{d \mathrm{x}^{l-k}}=0 \quad k=1,2, \ldots
\end{aligned}
$$

while equation (10) leads to

$$
\begin{aligned}
& \sum_{l=1}^{\infty} S_{l} \frac{d^{l} \mathrm{~V}^{(\mathrm{i})}}{d \mathrm{x}^{l}}=-4 \mathrm{~V}_{\mathrm{eff}}^{(2)} \\
& \sum_{l=2}^{\infty}\left(\begin{array}{l}
l \\
1
\end{array}\right) S_{l} \frac{d^{l-1} \mathrm{~V}^{(\mathrm{i})}}{d \mathrm{x}^{l-1}}=2 \frac{d \mathrm{M}^{(2)}}{d \mathrm{x}} \\
& \sum_{l=3}^{\infty}\left(\begin{array}{l}
l \\
2
\end{array}\right) S_{l} \frac{d^{l-2} \mathrm{~V}^{(\mathrm{i})}}{d \mathrm{x}^{l-2}}=2 \mathrm{M}^{(2)} \\
& \sum_{l=k+1}^{\infty}\left(\begin{array}{l}
l \\
k
\end{array}\right) S_{l} \frac{d^{l-k} \mathrm{~V}^{(\mathrm{i})}}{d \mathrm{x}^{l-k}}=0 \quad k=3,4, \ldots
\end{aligned}
$$

To be able to solve the general equations (15)-(20), it is appropriate to make some simplifying assumption. Inspired by the example of the $\mathcal{P} \mathcal{T}$-symmetric cubic anharmonic oscillator considered in [3, 4, where $Q_{1}$ only contains linear and cubic powers of p, let us assume that $R_{k}(\mathrm{x})=0, k=2,3, \ldots$, in equation (11). It then follows from (14) that only the first four functions $S_{k}$ in the expansion (13) may be nonvanishing and that they are given in terms of $R_{0}, R_{1}$, and their derivatives by $S_{0}=R_{0}^{\prime}-R_{1}^{\prime \prime \prime}, S_{1}=2 R_{0}-3 R_{1}^{\prime \prime}$, $S_{2}=-3 R_{1}^{\prime}$ and $S_{3}=-2 R_{1}$.

Let us first solve equations (15) and (16). In the latter, $k$ is now restricted to $k \leq 4$. For $k=4$, we obtain that $S_{3}$ must be a constant, this implying that

$$
R_{1}(\mathrm{x})=c_{1}
$$

Hence the remaining nonvanishing $S_{k}$ 's are

$$
S_{0}=R_{0}^{\prime} \quad S_{1}=2 R_{0} \quad S_{3}=-2 c_{1} .
$$


From equation (16) with $k=2$, we get

$$
R_{0}(\mathrm{x})=3 c_{1} \mathrm{~V}^{(\mathrm{r})}(\mathrm{x})+c_{0}
$$

where $c_{0}$ is another integration constant, while the equations with $k=1$ or $k=3$ are automatically satisfied. Equation (15) then provides us with a condition on $\mathrm{V}^{(\mathrm{i})}$,

$$
\mathrm{V}^{(\mathrm{i})}(\mathrm{x})=\frac{1}{4} c_{1} \mathrm{~V}^{(\mathrm{r}) \prime \prime \prime}(\mathrm{x})-\left[3 c_{1} \mathrm{~V}^{(\mathrm{r})}(\mathrm{x})+c_{0}\right] \mathrm{V}^{(\mathrm{r}) \prime}(\mathrm{x}) .
$$

Let us next turn ourselves to equations (17)-(20). It is easy to see that only equations (17) and (19) impose some new conditions, namely

$$
\mathrm{M}^{(2)}(\mathrm{x})=-3 c_{1} \mathrm{~V}^{(\mathrm{i}) \prime}(\mathrm{x}) \quad \mathrm{V}_{\mathrm{eff}}^{(2)}(\mathrm{x})=\frac{1}{2}\left\{-\left[3 c_{1} \mathrm{~V}^{(\mathrm{r})}(\mathrm{x})+c_{0}\right] \mathrm{V}^{(\mathrm{i}) \prime}(\mathrm{x})+c_{1} \mathrm{~V}^{(\mathrm{i}) \prime \prime \prime}(\mathrm{x})\right\}
$$

where $\mathrm{V}^{(\mathrm{i})}(\mathrm{x})$ must be expressed in terms of $\mathrm{V}^{(\mathrm{r})}(\mathrm{x})$ through equation (24). This completes the solution of equations (9) and (10).

It is then straightforward to go back to $x, p$ and unscaled operators. This leads to the conclusion that there exists a whole class of Hermitian PDM Hamiltonians, which to lowest order of perturbation theory allow an equivalent $\mathcal{P} \mathcal{T}$-symmetric description and might therefore be easier to deal with than generic ones. The various members of the class are distinguished by the choice of the zeroth-order part $V^{(\mathrm{r})}(x)$ of the effective potential $V_{\text {eff }}^{(2)}(x)$ and that of two integration constants $c_{0}, c_{1}$. The lowest-order corrections to the mass term $M^{(2)}(x)$ and to the effective potential in the PDM equation, as well as the imaginary part $V^{(i)}(x)$ of the corresponding $\mathcal{P} \mathcal{T}$-symmetric potential, are indeed entirely fixed by such a choice.

The classical Hamiltonians $H_{\mathrm{c}}\left(x_{\mathrm{c}}, p_{\mathrm{c}}\right)$ corresponding to the members of this class can be obtained by replacing $x$ and $p$ in $h$ by the classical variables $x_{\mathrm{c}}$ and $p_{\mathrm{c}}$ and evaluating the resulting expressions in the limit $\hbar \rightarrow 0$ (assuming this limit exists), i.e., $H_{\mathrm{c}}\left(x_{\mathrm{c}}, p_{\mathrm{c}}\right)=$ $\lim _{\hbar \rightarrow 0} h\left(x_{\mathrm{c}}, p_{\mathrm{c}}\right)$.

The $\eta_{+}$-pseudo-Hermitian position and momentum operators $X$ and $P$, as well as the physical wavefunctions $\Psi(x)$, can be calculated in the same way as $h$. To second order in $\varepsilon$, the pseudo-Hermitian operators are given by

$$
O=o-\frac{1}{2} \varepsilon\left[o, Q_{1}\right]+\frac{1}{8} \varepsilon^{2}\left[\left[o, Q_{1}\right], Q_{1}\right] \quad o=x \text { or } p .
$$


For the dimensionless operators, we find

$$
\begin{aligned}
& {\left[\mathrm{x}, Q_{1}\right]=\mathrm{i} \sum_{k=0}^{\infty}(k+1) S_{k+1} \frac{d^{k}}{d \mathrm{x}^{k}} \quad\left[\left[\mathrm{x}, Q_{1}\right], Q_{1}\right]=\sum_{k=0}^{\infty} T_{k} \frac{d^{k}}{d \mathrm{x}^{k}}} \\
& {\left[\mathrm{p}, Q_{1}\right]=-\sum_{k=0}^{\infty} \frac{d S_{k}}{d \mathrm{x}} \frac{d^{k}}{d \mathrm{x}^{k}} \quad\left[\left[\mathrm{p}, Q_{1}\right], Q_{1}\right]=\mathrm{i} \sum_{k=0}^{\infty} U_{k} \frac{d^{k}}{d \mathrm{x}^{k}}}
\end{aligned}
$$

where $T_{k}$ and $U_{k}$ are defined by

$$
T_{k}=\sum_{l=0}^{k} \sum_{m=k-l+1}^{\infty} T_{k}^{(l, m)} \quad U_{k}=\sum_{l=0}^{k} \sum_{m=k-l+1}^{\infty} U_{k}^{(l, m)}
$$

with

$$
\begin{aligned}
T_{k}^{(l, m)} & =\left(\begin{array}{c}
m \\
k-l
\end{array}\right)\left[(m+1) S_{m+1} \frac{d^{l+m-k} S_{l}}{d \mathrm{x}^{l+m-k}}-(l+1) S_{m} \frac{d^{l+m-k} S_{l+1}}{d \mathrm{x}^{l+m-k}}\right] \\
U_{k}^{(l, m)} & =\left(\begin{array}{c}
m \\
k-l
\end{array}\right)\left[\frac{d S_{m}}{d \mathrm{x}} \frac{d^{l+m-k} S_{l}}{d \mathrm{x}^{l+m-k}}-S_{m} \frac{d^{l+m-k+1} S_{l}}{d \mathrm{x}^{l+m-k+1}}\right] .
\end{aligned}
$$

Similarly, the physical wavefunctions can be expressed as

$$
\Psi(\mathrm{x})=\psi(x)-\frac{\varepsilon}{2}\left\langle\mathrm{x}\left|Q_{1}\right| \psi\right\rangle+\frac{\varepsilon^{2}}{8}\left\langle\mathrm{x}\left|Q_{1}^{2}\right| \psi\right\rangle
$$

where $Q_{1}$ is given by (13) and

$$
Q_{1}^{2}=-\sum_{k=0}^{\infty} W_{k}(\mathrm{x}) \frac{d^{k}}{d \mathrm{x}^{k}}
$$

with

$$
W_{k}=\sum_{l=0}^{k} \sum_{m=k-l}^{\infty} W_{k}^{(l, m)} \quad W_{k}^{(l, m)}=\left(\begin{array}{c}
m \\
k-l
\end{array}\right) S_{m} \frac{d^{l+m-k} S_{l}}{d \mathrm{x}^{l+m-k}} .
$$

With the simplifying assumption (22) and taking equations (23) and (26) -(32) into account, we obtain

$$
\begin{aligned}
\mathrm{X}= & \mathrm{x} \\
& -\mathrm{i} \varepsilon\left(3 c_{1} \mathrm{~V}^{(\mathrm{r})}+c_{0}+3 c_{1} \mathrm{p}^{2}\right)+\frac{3}{4} \varepsilon^{2} c_{1}\left[-c_{1}\left(6 \mathrm{~V}^{(\mathrm{r})} \mathrm{V}^{(\mathrm{r}) \prime}+\mathrm{V}^{(\mathrm{r}) \prime \prime \prime}\right)-2 c_{0} \mathrm{~V}^{(\mathrm{r}) \prime} \mathrm{p}+6 c_{1} \mathrm{~V}^{(\mathrm{r}) \prime} \mathrm{p}^{2}\right] \\
\mathrm{P}=\mathrm{p} & +\frac{3}{2} \varepsilon c_{1}\left(\mathrm{~V}^{(\mathrm{r}) \prime \prime}+2 \mathrm{i} \mathrm{V}^{(\mathrm{r}) \prime} \mathrm{p}\right)+\frac{3}{4} \mathrm{i} \varepsilon^{2} c_{1}\left\{c_{1}\left(3 \mathrm{~V}^{(\mathrm{r}) \prime} \mathrm{V}^{(\mathrm{r}) \prime \prime}-3 \mathrm{~V}^{(\mathrm{r})} \mathrm{V}^{(\mathrm{r}) \prime \prime \prime}+\mathrm{V}^{(\mathrm{r}) \prime \prime \prime \prime \prime \prime}\right)\right. \\
& -c_{0} V^{(\mathrm{r}) \prime \prime \prime}+\mathrm{i}\left[c_{1}\left(6 \mathrm{~V}^{(\mathrm{r}) \prime 2}-6 \mathrm{~V}^{(\mathrm{r})} \mathrm{V}^{(\mathrm{r}) \prime \prime}+5 \mathrm{~V}^{(\mathrm{r}) \prime \prime \prime \prime}\right)-2 c_{0} \mathrm{~V}^{(\mathrm{r}) \prime \prime}\right] \mathrm{p}-9 c_{1} \mathrm{~V}^{(\mathrm{r}) \prime \prime \prime} \mathrm{p}^{2} \\
& \left.-6 \mathrm{i} c_{1} \mathrm{~V}^{(\mathrm{r}) \prime \prime} \mathrm{p}^{3}\right\}
\end{aligned}
$$


and

$$
\begin{aligned}
\Psi(\mathrm{x})= & \psi(x)+\frac{1}{2} \mathrm{i} \varepsilon\left[3 c_{1} \mathrm{~V}^{(\mathrm{r}) \prime}+2\left(3 c_{1} \mathrm{~V}^{(\mathrm{r})}+c_{0}\right) \frac{d}{d \mathrm{x}}-2 c_{1} \frac{d^{3}}{d \mathrm{x}^{3}}\right] \\
& -\frac{\varepsilon^{2}}{8}\left\{3 c_{1}\left[c_{1}\left(3 \mathrm{~V}^{(\mathrm{r}) \prime 2}+6 \mathrm{~V}^{(\mathrm{r})} \mathrm{V}^{(\mathrm{r}) \prime \prime}-2 \mathrm{~V}^{(\mathrm{r}) \prime \prime \prime \prime}\right)+2 c_{0} \mathrm{~V}^{(\mathrm{r}) \prime \prime}\right]\right. \\
& +6 c_{1}\left[c_{1}\left(12 \mathrm{~V}^{(\mathrm{r})} \mathrm{V}^{(r) \prime}-5 \mathrm{~V}^{(\mathrm{r}) \prime \prime \prime}\right)+4 c_{0} \mathrm{~V}^{(r) \prime}\right] \frac{d}{d \mathrm{x}}+2\left[9 c_{1}^{2}\left(2 \mathrm{~V}^{(\mathrm{r}) 2}-3 \mathrm{~V}^{(\mathrm{r}) \prime \prime}\right)\right. \\
& \left.+12 c_{0} c_{1} \mathrm{~V}^{(\mathrm{r})}+2 c_{0}^{2}\right] \frac{d^{2}}{d \mathrm{x}^{2}}-48 c_{1}^{2} \mathrm{~V}^{(\mathrm{r}) \prime} \frac{d^{3}}{d \mathrm{x}^{3}}-8 c_{1}\left(3 c_{1} \mathrm{~V}^{(\mathrm{r})}+c_{0}\right) \frac{d^{4}}{d \mathrm{x}^{4}} \\
& \left.+4 c_{1}^{2} \frac{d^{6}}{d \mathrm{x}^{6}}\right\}
\end{aligned}
$$

It is easy to check that, as expected, the Hermitian PDM quartic anharmonic oscillator of 3, 4] belongs to the class of Hermitian PDM Hamiltonians with an approximate $\mathcal{P} \mathcal{T}$ symmetric counterpart. On setting $\mathrm{V}^{(\mathrm{r})}(\mathrm{x})=\frac{1}{2} \mathcal{M}^{2} \mathrm{x}^{2}, c_{0}=0$ and $c_{1}=-2 /\left(3 \mathcal{M}^{4}\right)$ in equation (24), where the dimensionless quantitites are defined as in equations (17)-(20) of [4], we indeed obtain $\mathrm{V}^{(\mathrm{i})}(\mathrm{x})=\mathrm{x}^{3}$, so that $V^{(\mathrm{i})}(x)=x^{3}$. Furthermore, from equations (25), (33) and (34), we obtain $m(x)=m_{0}\left[1+6\left(\epsilon^{2} / \mu^{4}\right) x^{2}\right]^{-1}, V_{\mathrm{eff}}^{(2)}(x)=\left(3 m_{0} \mu^{2} x^{4}-4 \hbar^{2}\right) /\left(2 m_{0} \mu^{4}\right)$, $X=x+\mathrm{i}\left(\epsilon / \mu^{4}\right)\left(\mu^{2} x^{2}+2 p^{2} / m_{0}\right)+\left(\epsilon^{2} / \mu^{6}\right)\left(-\mu^{2} x^{3}-2 \mathrm{i} \hbar p / m_{0}+2 x p^{2} / m_{0}\right)$ and $P=$ $p-\mathrm{i}\left(\epsilon / \mu^{2}\right)(2 x p-\mathrm{i} \hbar)+\left(\epsilon^{2} / \mu^{6}\right)\left(2 p^{3} / m_{0}-\mu^{2} x^{2} p+\mathrm{i} \hbar \mu^{2} x\right)$, which after some reordering agree with [3, 4], as does the classical Hamiltonian. Similarly, equation (35) gives rise to equation (65) of 4 .

A new example is provided by selecting for $V^{(\mathrm{r})}(x)$ a one-parameter trigonometric Pöschl-Teller potential [15]

$$
V^{(\mathrm{r})}(x)=V_{0} \sec ^{2} k x \quad V_{0}=\frac{\hbar^{2} k^{2}}{2 m^{2}} \lambda(\lambda-1) \quad \lambda>2
$$

on the interval $-\frac{\pi}{2} \leq x \leq \frac{\pi}{2}$. On setting $\ell=k^{-1}$ and $\nu=\hbar^{2} k^{2} / m_{0}$ for the length and energy scales, respectively, we obtain the dimensionless quantities $\mathrm{x}=k x, \mathrm{p}=p /(\hbar k)$ and $\mathrm{V}^{(\mathrm{r})}(\mathrm{x})=\frac{1}{2} \lambda(\lambda-1) \sec ^{2} \mathrm{x}$ with $\lambda(\lambda-1)=2 V_{0} / \nu$.

The choice $c_{0}=-c_{1}=\frac{1}{3}$ in (24) leads to

$$
\mathrm{V}^{(\mathrm{i})}(\mathrm{x})=\frac{1}{2}(\lambda+1) \lambda(\lambda-1)(\lambda-2) \sec ^{4} \mathrm{x} \tan \mathrm{x}=\frac{2}{\nu^{2}} V_{0}\left(V_{0}-\nu\right) \sec ^{4} \mathrm{x} \tan \mathrm{x} .
$$


This means that the corresponding $\mathcal{P} \mathcal{T}$-symmetric Hamiltonian may be written as

$$
H=\frac{p^{2}}{2 m_{0}}+V_{0} \sec ^{2} k x+\mathrm{i} \epsilon \sec ^{4} k x \tan k x
$$

where $\epsilon$ has the dimension of an energy and is given in terms of the dimensionless $\varepsilon$ by $\epsilon=2 \varepsilon V_{0}\left(V_{0}-\nu\right) / \nu$.

To second order in $\epsilon$, such a non-Hermitian Hamiltonian is equivalent to a Hermitian $\mathrm{PDM}$ one, given by equation (5), where

$$
m(x)=m_{0}\left(1+\frac{\epsilon^{2}}{2 V_{0}\left(V_{0}-\nu\right)} \sec ^{4} k x\left(5 \sec ^{2} k x-4\right)\right)^{-1}
$$

and

$$
V_{\text {eff }}(x)=V_{0} \sec ^{2} k x+\frac{\epsilon^{2}}{4 V_{0}\left(V_{0}-\nu\right)} \sec ^{4} k x\left[5\left(V_{0}-14 \nu\right) \sec ^{4} k x-\left(4 V_{0}-85 \nu\right) \sec ^{2} k x-20 \nu\right] .
$$

The corresponding $\eta_{+}$-pseudo-Hermitian position and momentum operators can be expressed as

$$
\begin{aligned}
X= & x-\mathrm{i} \frac{\epsilon}{2 k V_{0}\left(V_{0}-\nu\right)}\left(-V_{0} \sec ^{2} k x+\frac{\nu}{3}-\frac{p^{2}}{m_{0}}\right)-\frac{\epsilon^{2}}{4 k V_{0}\left(V_{0}-\nu\right)^{2}} \sec ^{2} k x \\
& \times\left\{\left[\left(V_{0}+2 \nu\right) \sec ^{2} k x-\nu\right] \tan k x+\mathrm{i} \sqrt{\frac{\nu}{m_{0}}}\left(3 \sec ^{2} k x-2\right) p-\tan k x \frac{p^{2}}{m_{0}}\right\} \\
P= & -\frac{\epsilon}{2\left(V_{0}-\nu\right)} \sec ^{2} k x\left[\sqrt{m_{0} \nu}\left(3 \sec ^{2} k x-2\right)+2 \mathrm{i} \tan k x p\right]-\mathrm{i} \frac{\epsilon^{2}}{4 V_{0}\left(V_{0}-\nu\right)^{2}} \sec ^{2} k x \\
& \times\left\{\sqrt{m_{0} \nu}\left[3 V_{0} \sec ^{4} k x-2 \nu\left(30 \sec ^{4} k x-19 \sec ^{2} k x+1\right)\right]+\mathrm{i}\left[V_{0} \sec ^{4} k x\right.\right. \\
& \left.-\nu\left(50 \sec ^{4} k x-49 \sec ^{2} k x+6\right)\right] p+6 \sqrt{\frac{\nu}{m_{0}}}\left(3 \sec ^{2} k x-1\right) \tan k x p^{2} \\
& \left.+\frac{\mathrm{i}}{m_{0}}\left(3 \sec ^{2} k x-2\right) p^{3}\right\} .
\end{aligned}
$$

Similar results can be found for physical wavefunctions. For lack of space, let us only mention the result in dimensionless variable obtained for the function $\psi(\mathrm{x})=\cos ^{\lambda}(\mathrm{x})$ (corresponding to the ground state of the real potential (36)):

$$
\begin{aligned}
\Psi(\mathrm{x})= & \cos ^{\lambda}(\mathrm{x})\left\{1+\frac{\mathrm{i}}{6} \varepsilon(\lambda+1) \lambda(\lambda-1)\left(\sec ^{2} \mathrm{x}+2\right) \tan \mathrm{x}-\frac{\varepsilon^{2}}{72}(\lambda+1) \lambda(\lambda-1)\right. \\
& \times\left[(\lambda-4)(\lambda-2)(\lambda+15) \sec ^{6} \mathrm{x}+3(\lambda-2)\left(\lambda^{2}-4 \lambda+15\right) \sec ^{4} \mathrm{x}\right. \\
& -4(\lambda+1) \lambda(\lambda-1)\} .
\end{aligned}
$$


In the classical limit, $\nu$ goes to zero. To get a nonvanishing limit for $V_{0}$, we must therefore assume that $\lambda$ goes to infinity as $\hbar^{-1}$ (this implying, in particular, that $\lambda$ becomes negligeably small compared with $\lambda^{2}$ ). To second order in $\epsilon$, the classical Hamiltonian corresponding to (38) is obtained as

$$
H_{\mathrm{c}}=\frac{p_{\mathrm{c}}^{2}}{2 m\left(x_{\mathrm{c}}\right)}+V_{0} \sec ^{2} k x_{\mathrm{c}}+\frac{\epsilon^{2}}{4 V_{0}} \sec ^{6} k x_{\mathrm{c}}\left(5 \sec ^{2} k x_{\mathrm{c}}-4\right)
$$

where

$$
m_{\mathrm{c}}\left(x_{\mathrm{c}}\right)=m_{0}\left(1-\frac{\epsilon^{2}}{2 V_{0}^{2}} \sec ^{4} k x_{\mathrm{c}}\left(5 \sec ^{2} k x_{\mathrm{c}}-4\right)\right)
$$

while the classical $\eta_{+}$-pseudo-Hermitian variables $X_{\mathrm{c}}, P_{\mathrm{c}}$ are

$$
\begin{aligned}
X_{\mathrm{c}}= & x_{\mathrm{c}}+\mathrm{i} \frac{\epsilon}{2 k V_{0}^{2}}\left(V_{0} \sec ^{2} k x_{\mathrm{c}}+\frac{p_{\mathrm{c}}^{2}}{m_{0}}\right)-\frac{\epsilon^{2}}{4 k V_{0}^{3}} \sec ^{2} k x_{\mathrm{c}} \\
& \times\left(V_{0} \sec ^{2} k x_{\mathrm{c}}-\frac{p_{\mathrm{c}}^{2}}{m_{0}}\right) \tan k x_{\mathrm{c}} \\
P_{\mathrm{c}}= & p_{\mathrm{c}}-\mathrm{i} \frac{\epsilon}{V_{0}} \sec ^{2} k x_{\mathrm{c}} \tan k x_{\mathrm{c}} p_{\mathrm{c}}+\frac{\epsilon^{2}}{4 V_{0}^{3}} \sec ^{2} k x_{\mathrm{c}} \\
& \times\left[V_{0} \sec ^{4} k x_{\mathrm{c}}+\left(3 \sec ^{2} k x_{\mathrm{c}}-2\right) \frac{p_{\mathrm{c}}^{2}}{m_{0}}\right] p_{\mathrm{c}} .
\end{aligned}
$$

It is worth noting that in contrast with what happens for the $\mathcal{P} \mathcal{T}$-symmetric cubic anharmonic oscillator, the operators $X$ and $P$ involve $\hbar$ even after rewritting them in a symmetrized form. As a consequence, the $\eta_{+}$-pseudo-Hermitian quantization of the classical Hamiltonian (44) is far from trivial. This illustrates the importance of the factor-ordering problem in pseudo-Hermitian quantum mechanics.

In conclusion, the generalization of the works in [3] and [4] that we have proposed here contributes to exploring further the relationships between $\mathcal{P} \mathcal{T}$-symmetric and Hermitian PDM Hamiltonians started there and continued in [7, 16]. Moreover, it suggests the interest

of performing detailed calculations for some new $\mathcal{P} \mathcal{T}$-symmetric systems, such as the one defined in (38).

\section{Acknowledgments}

BB and RR gratefully acknowledge the support of the National Fund for Scientific Research (FNRS), Belgium, and the warm hospitality at PNTPM, Université Libre de Bruxelles, 
where this work was initiated. CQ is a Research Director of the National Fund for Scientific Research (FNRS), Belgium. 


\section{References}

[1] Bender C M and Boettcher S 1998 Phys. Rev. Lett. 805243

[2] Mostafazadeh A 2002 J. Math. Phys. 43205

Mostafazadeh A 2002 J. Math. Phys. 432814

Mostafazadeh A and Batal A 2004 J. Phys. A: Math. Gen. 3711645

[3] Jones H F 2005 J. Phys. A: Math. Gen. 381741

[4] Mostafazadeh A 2005 J. Phys. A: Math. Gen. 38 6557, 8185

[5] Dorey P, Dunning C and Tateo R 2001 J. Phys. A: Math. Gen. 345679

Shin K C 2002 Commun. Math. Phys. 229543

[6] Bender C M, Meisinger P N and Wang Q 2003 J. Phys. A: Math. Gen. 361973

Bender C M, Brody D C and Jones H F 2004 Phys. Rev. D 70025001

Bender C M, Brod J, Refig A and Reuter M E 2004 J. Phys. A: Math. Gen. 3710139

[7] Bender C M, Chen J-H and Milton K A $2005 \mathcal{P} \mathcal{T}$-symmetric versus Hermitian formulations of quantum mechanics Preprint hep-th/0511229

[8] Bagchi B, Gorain P, Quesne C and Roychoudhury R 2004 Mod. Phys. Lett. A 192765

[9] Koç R and Koca M 2003 J. Phys. A: Math. Gen. 368105

[10] Roy B and Roy P 2005 J. Phys. A: Math. Gen. 3811019

[11] Gönül B and Koçak M 2005 Chin. Phys. Lett. 202742

[12] Bagchi B and Quesne C 2000 Phys. Lett. A 273285

[13] Ahmed Z 2001 Phys. Lett. A 29019

[14] von Roos O 1983 Phys. Rev. B 277547

[15] Nieto M M 1978 Phys. Rev. A 171273

Quesne C 1999 J. Phys. A: Math. Gen. 326705 
[16] Bagchi B, Quesne C and Roychoudhury R 2005 J. Phys. A: Math. Gen. 38 L647 